\title{
Observation of "Integration of Breath, Blood and Voice" Acupoint Control Singing to Rehabilitate Senile Dementia and Degenerative Deafness Cases
}

\author{
Zhao Ling ${ }^{1,2}$ \\ 1. Department of Music, Jiaozuo Normal College, Jiaozuo, Henan 454000, China \\ 2. Zhao Ling TCM Meridian Musical Rehabilitation Guidance Department, Jiaozuo, Henan 454000, China
}

\begin{abstract}
PURPOSE: To observe the treat of Acupuncture point control singing rehabilitating senile dementia and degenerative deafness. TECHNIQUE: Utilizing the method of "Integration of Breath, Blood and Voice" Acupoint Control Singing to Rehabilitate Senile Dementia and Degenerative Deafness. CONSEQUENCE: After Four courses of treatment, the patients of senile dementia are able to talk and basically take care of themselves. Only one hour of practice lead the patients with mild senile dementia to remove the audiphone and sing successfully. The elderly patients with degenerative deafness in the two courses of treatment showed significant improvement with language recognition rate. CONCLUSION: This high-energy and low consumption comprehensive method has a good effect for the rehabilitation of the patients with senile dementia and senile degenerative deafness.
\end{abstract}

Key words: Acupoint control singing, moving and positioning stimulation, senile dementia, senile degenerative deafness.

In this paper, patients with mild and severe senile dementia ( 2 cases) and senile degenerative deafness ( 3 cases) were observed for the case of rehabilitation, under the cure of moving and positioning stimulation combined with acupoint control singing for 1 hour/1 day $/ 5$ days/1 course of treatment in "Integration of Breath, Blood and Voice" method [1].

\section{Methods of Rehabilitation}

Utilize the methods and requirements of moving and positioning needle acupuncture stimulation in conjunction with singing pronunciation.

The methods and requirements of moving needle acupuncture stimulation and singing pronunciation.

According to the way of pronunciation of singing, practice in an ascending and descending way for the 1-6 pronunciation exercises, with the method of moving needle acupuncture manipulation (bamboo needle) in order to stimulate the acupuncture point, add

Corresponding author: Zhao Ling, B.S., professor, acupuncture doctor, research field: music teaching and meridian music rehabilitation therapy. melody practice after two rounds [2].

\subsection{Pronunciation Practice of Singing}

Practice in grading, skipping, staccato, legato, arpeggio ways [2].

(Artical.1)

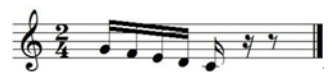

\section{(Artical.1)}

$$
\text { mi mi mi mi mi }
$$

(Artical.2) mi

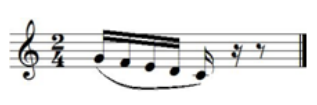

(Artical.3)

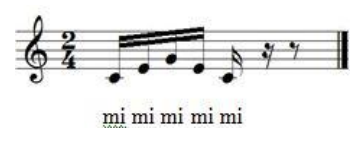

(Artical.4)

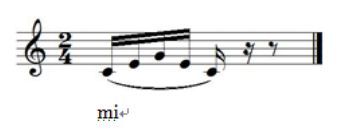

(Artical.5)

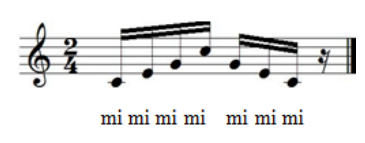

(Artical.6)

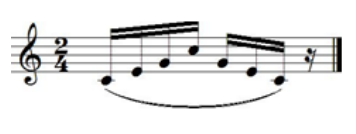


1.2 The Requirements of Moving Needle Acupuncture Points Matching Pronunciation Practice of Singing

(1) Stimulate the No.1 acupuncture points on both sides of mouth when practicing the Article 1.

(2) Stimulate the No.8 acupuncture points on both sides of mouth when practicing the Article 2.

(3) Stimulate the No.2 acupuncture points on both sides of nose when practicing the Article 3 .

(4) Stimulate the No.8 acupuncture points in the central on both sides of the nose when practicing the Article 4.

(5) Stimulate the No.14 acupuncture points on the root of the nose when practicing the Article 5.

(6) Stimulate the No.1 acupuncture points on both sides of eyes when practicing the Article 6 .

(7) Stimulate the No.7 acupuncture points on both sides of the forehead when practicing the Article 5.

(8) Stimulate the No.11 acupuncture points on both sides of the forehead when practicing the Article 6 .

(9) Stimulate the No.7 acupuncture points on both sides of the forehead when practicing "The butterfly lovers". The intensity of acupuncture point stimulation should be controlled with the pronunciation of the sound level and the speed of rhythm, stop stimulating when breathing in [2].

\subsection{The Methods and Requirements of Positioning Needle Acupuncture Stimulation with Moving Needle Acupuncture and Singing Pronunciation}

Apply positioning needle when practicing the vocalise in the order of 1-8 at the first and second courses of treatment [2]. Add the 9th practicing of "The butterfly lovers" after the second course of treatment.

(1) Positioning needle acupuncture point stimulation for severe senile dementia: The No.1, No.2, No.4, No.5, No.7, No.8 acupuncture points on the top of head (9 acupuncture points). Practice the vocalise in the order of 1-8 and add No.9 in the third and forth courses of treatment, the acupuncture points of positioning needle stimulation should be: The No.1, No.2, No.4, No.10 acupuncture points on the top of head (5 acupuncture points), the No.2, No.5 acupuncture points located the upper part at the back of the neck (four acupuncture points). Keep stimulating the acupuncture points for a period of time after the singing exercise [3].

(2) Positioning needle acupuncture stimulation is not mild senile dementia patients' choice. These patients should be rehabilitated only use moving needle acupuncture stimulation and singing practice [2].

(3) Senile degenerative deafness patients should be treated with positioning needle acupuncture stimulation: the acupuncture points on the top of head are the same as the severe senile dementia patients, the No.21, No. 22, No. 23 acupuncture points on the cheek (the front of the ear) [2].

\section{Rehabilitation Case-Senile Dementia and Degenerative Deafness [1]}

\subsection{Severely Senile Dementia (1 Case)}

Zhang Qidong, male, 74 years old. The onset of the disease started 13 years ago. The patient diagnosed by the hospital as cerebellum atrophy; brain stem chronic infarction; moderate senile dementia. The diagnosis of the hospital, CT indicated cerebral white matter degeneration and demyelination; multiple lacunar infarction; cerebral atrophy; severe senile dementia. Performance with confusion, speechless, expressionless, slobber, incapable of dressing and undressing, cannot self-care with defecation, no cognition with the surroundings, no self control with emotion, performance with depression.

\subsection{Mid Senile Dementia (1 Case)}

Gao Fuming, male, 87 years old, a retired opera singer. Wearing audiphone due to the mild senile dementia and senile degenerative deafness, speak slowly and low, can only pronounce in a flat way when read "The lovely a Rose", drag every single word long with no tone and rhythm, incapable of singing this song with rhythm and a high or low pitch. 


\subsection{Senile Degenerative Deafness (3 Cases)}

Case 1. Jing Dianyu, female, 73 years old. Medical history for 5 years. The diagnosis from the hospital indicated severe senile degenerative deafness.

Case 2. Liu Chunlan, female, 64 years old. Medical history for 5 years. The diagnosis from the hospital indicated extremely severe senile degenerative deafness.

Case 3. Li Xiuzhen, female, 70 years old. Medical history for 5 years. The diagnosis from the hospital indicated mid senile degenerative deafness [1].

\section{Rehabilitation Efficacy}

\subsection{Severely Senile Dementia (1 Case) and Mid Senile Dementia (1 Case)}

Zhang Qidong, after four causes of treatment for the patient with severe senile dementia: the patient can think clearly, participate in multi-people conversation, talk fluently, the symptoms of stroke disappeared, can basically take care of themselves.

Gao Fuming, after practice only once/1 hour for the patient with mild senile dementia: the patients can live without the audiphones and able to sing the "The Lovely a Rose" completely, rhythm, and a high or low pitch immediately [1].

\subsection{Rehabilitation Efficacy for Senile Degenerated Deafness after Two Courses of Treatment}

Jing Dianyu, the language recognition rate score of Jing Dianyu was $95 \% / 80 \mathrm{~dB}$ before the rehabilitation, the score up to $96 \% / 75 \mathrm{~dB}$ after the first course of treatment; then reached $96 \% / 70 \mathrm{~dB}$ after the second treatment.
Liu Chunlan, the language recognition rate score of Liu Chunlan was $75 \% / 95 \mathrm{~dB}$ before the rehabilitation, the score up to $88 \% / 90 \mathrm{~dB}$ after the first course of treatment; the score reached $88 \% / 85 \mathrm{~dB}$ after the second treatment.

Li Xiuzhen, the language recognition rate score of $\mathrm{Li}$ Xiuzhen was $90 \% / 70 \mathrm{~dB}$ before the rehabilitation, the score up to $96 \% / 70 \mathrm{~dB}$ after the first course of treatment; the score reached $100 \% / 70 \mathrm{~dB}$ after the second treatment.

\section{Conclusion}

With the method of stimulation of acupuncture point in conjunction with "Integration of Breath, Blood and Voice" [1] acupoint control singing can bring the senile dementia and degenerative deafness [4] patient's great rehabilitation efficacy. The comprehensive function and multi effect of acupoint control "Integration of Breath, Blood and Voice" method, and the effect of the whole system of bidirectional regulation of comprehensive energy formation strengthening, including the scientific and standardized form of rehabilitation [2] with the utilize of fixed-point, positioning, qualitative and quantitative, are all the fundamental reasons that the patients obtain significant rehabilitation efficacy in a short period of time.

\section{Reference}

[1] Zhao, L. 2016. Singing Meridian Theory Practice Research (DE). Saarbrucken: Golden Light Academic Press.

[2] Zhao, L. 2014. Singing Meridian Study. Shenyang: Northeastern University press.

[3] Liu, G. J. 1987. Acupuncture Science. Hunan. Changsha: Hunan Science and Technology Publishing House.

[4] Nong, H. T. 2011. Otolaryngology Head and Neck Surgery. Beijing: People's Medical Publishing House. 